DOI: $10.24850 /$ j-tyca-2020-06-09

Notas

\title{
Adecuación para consumo humano de propiedades físicas de aguas del río Carrizal, Ecuador, a través de microorganismos eficientes y filtración con zeolitas Adaptation for human consumption of physical properties of Carrizal River waters, Ecuador, through efficient microorganisms and filtration with zeolites
}

Ángela Carreño1, ORCID: https://orcid.org/0000-0001-5661-0906

Leonel Lucas², ORCID: https://orcid.org/0000-0002-2286-9487

Ernesto Hurtado3, ORCID: https://orcid.org/0000-0003-2574-1289

Renny Barrios4, ORCID: https://orcid.org/0000-0002-7094-7115

Ramón Silva5, ORCID: https://orcid.org/0000-0003-1235-9283

${ }^{1}$ Carrera de Ingeniería Ambiental, Escuela Superior Politécnica Agropecuaria de Manabí, Calceta, Manabí, Ecuador, a.lore.carreno@gmail.com

${ }^{2}$ Carrera de Ingeniería Ambiental, Escuela Superior Politécnica Agropecuaria de Manabí, Calceta, Manabí, Ecuador; Universidad Espíritu Santo, Ecuador, leonel.luvi@gmail.com 
${ }^{3}$ Carrera de Medicina Veterinaria, Escuela Superior Politécnica Agropecuaria de Manabí, Calceta, Manabí, Ecuador, ernestohurta@gmail.com

${ }^{4}$ Instituto Nacional de Investigaciones Agropecuarias, San Agustín de la Pica, Vía Laguna Grande, Maturín, Venezuela, rennybarrios@gmail.com ${ }^{5}$ Postgrado de Agricultura Tropical, Universidad de Oriente Campus Juanico, Maturín, Monagas, Venezuela,drramonsilvaa@gmail.com

Autor para correspondencia: Ernesto Antonio Hurtado, ernestohurta@gmail.com

\section{Resumen}

Con el objetivo de evaluar las características físicas de aguas para consumo humano, procedentes de las localidades de Severino, Julián y Balsa en Medio, ubicadas en la microcuenca del río Carrizal, Ecuador, tratadas por estrategias de depuración, se evaluaron cinco tratamientos: incubada con $5 \mathrm{ml} / \mathrm{l}$ de $\mathrm{EM} \bullet 1{ }^{\circledR}$ y filtrada en zeolita cubana; incubada con $5 \mathrm{ml} / \mathrm{l}$ de $\mathrm{EM} \bullet 1^{\circledR}$ y filtrada en zeolita ecuatoriana; e incubada con tres dosis de microorganismos autóctonos (5, 10 y $15 \mathrm{ml} / \mathrm{l})$. Se utilizó el diseño experimental de bloques al azar, con cuatro repeticiones. Las comparaciones se llevaron a cabo a través de contrastes ortogonales. Se constató que, con independencia de la localidad y la fuente de agua muestreada, se encontraron ventajas estadísticamente significativas ( $p<$ 0.05) para las variables turbidez, sólidos totales y dureza cuando se emplean dosis de $15 \mathrm{ml} / \mathrm{l}$ microorganismos autóctonos. Se obtuvieron 
resultados similares cuando se utilizó zeolita ecuatoriana como elemento filtrante asociado con microorganismos eficientes $\left(E M \bullet 1^{\circledR}\right)$. La variable temperatura presentó mayores valores relacionados con los tratamientos con los organismos eficientes $\left(\mathrm{EM} \bullet 1^{\circledR}\right)$, cuando son filtrados con la zeolita cubana y, de manera similar, con la dosis de $5 \mathrm{ml} / \mathrm{l}$ de microrganismos autóctonos. La variable color fue similar para todas las fuentes de muestreo y localidades. La mayoría de las variables físicas de las localidades de Severino, Julián y Balsa en Medio cumplen con las normativas aplicables a condiciones de calidad de agua de consumo de Ecuador. La turbidez excedió los límites permisibles y no fue posible corregirlos con los tratamientos evaluados.

Palabras clave: turbidez, sólidos totales, dureza, temperatura, color.

\section{Abstract}

With the objective of evaluating physical characteristics of waters for human consumption from the Severino, Julián and Balsa en Medio localities located in the micro-basin of the Carrizal River, Ecuador, treated by purification strategies, five treatments were evaluated: Incubated with $5 \mathrm{ml} / \mathrm{l}$ of $\mathrm{EM} \bullet 1^{\circledR}$ and filtered in Cuban zeolite; Incubated with $5 \mathrm{ml} / \mathrm{l}$ of $\mathrm{EM} \bullet 1^{\circledR}$ and filtered in Ecuadorian zeolite; Incubated with three doses of autochthonous microorganisms $(5,10$ and $15 \mathrm{ml} / \mathrm{l})$. The experimental design was completely randomized blocks, with four repetitions. Comparisons of means were made through orthogonal contrasts. It was found that regardless of the location and source of water sampled, statistically significant advantages were found for the variables turbidity, total solids and hardness when using doses of $15 \mathrm{ml} / \mathrm{l}$ autochthonous 
microorganisms. Similar results were obtained when the Ecuadorian zeolite associated with efficient microorganisms was used as filter element $\left(E M \bullet 1^{\circledR}\right)$. Temperature presented higher values associated with the treatments with efficient organisms $\left(E M \bullet 1^{\circledR}\right)$ when they were filtered with the Cuban zeolite and similarly with the dose of $5 \mathrm{ml} / \mathrm{l}$ of autochthonous microoganisms. The variable color was similar for all sampling sources and locations. Most of the physical variables of the Severino, Julián and Balsa en Medio localities meet with regulations of quality of drinking water of Ecuador. The turbidity exceeded the permissible limits and it was not possible to correct them with the evaluated treatments.

Keywords: Turbidity, total solids, hardness, temperature, color.

Recibido: $12 / 10 / 2018$

Aceptado: $26 / 02 / 2020$

\section{Introducción}

En las últimas décadas se han desarrollado nuevos e innovadores tratamientos de agua de uso doméstico, para llevar soluciones sencillas de bajo costo y fáciles de usar por personas que deben dar tratamiento al agua en sus hogares. Algunos de los sistemas domésticos utilizados 
con frecuencia para el tratamiento de agua son la cloración, filtración, desinfección solar, filtración/cloración combinadas, floculación/cloración combinadas y recientemente, el tratamiento con microorganismos eficientes (Global Health and Education Foundation, National Academy of Science, 2007).

En la provisión de agua se debe tener especial cuidado con el sabor, olor, color y turbidez del agua. El sabor y olor se deben a la presencia de sustancias químicas volátiles y a la materia orgánica en descomposición; mientras que el color es por la presencia de minerales como hierro y manganeso, materia orgánica y residuos industriales. El color en el agua doméstica puede manchar los accesorios sanitarios y opacar la ropa; por otro lado, la turbidez, además de que es objetable desde el punto de vista estético, puede contener agentes patógenos adheridos a las partículas en suspensión, como es el caso de arcillas (Orellana, 2005).

Se han adoptado diversas estrategias para mejorar la calidad del agua, entre ellas, las zeolitas que han sido probadas y reconocidas como los materiales naturales más eficientes para el mejoramiento de la calidad de agua, entre otros usos; además, es un mineral amigable con la naturaleza, químicamente inocua, no contamina y no sufre alteraciones (Márquez, Herrera, \& Gutiérrez, 2000). La zeolita, como un mineral de origen volcánico de gran estabilidad, alta capacidad de intercambio iónico y absorción de agua, así como de fácil extracción y transporte, lo hace muy útil (Paredes, 2014). Este material presenta una red tridimensional de unidades tetrahédricas de óxidos de silicio y aluminio, unidas por un átomo de oxígeno compartido. El reemplazo isomorfo de $\mathrm{Si}^{4+}$ por $\mathrm{Al}^{3+}$ resulta en una carga negativa global compensada por una base o cationes 
metálicos basificados del medio (Covarrubias García, Yánez, \& Arriagada, 2008; Wang \& Peng, 2010).

Diversas investigaciones indican el uso potencial de la zeolita en procesos de filtración de aguas para consumo humano (Wang et al., 2010; Mishué, Hernández, \& González, 2012; Cuchimaque, Vargas, \& Ríos, 2013; Vera, Rojas, Chávez, \& Arriaza 2016). Por otro lado, el uso de microorganismos eficientes, representados por la combinación de microorganismos benéficos naturales de los géneros Lactobacillus (bacterias ácido lácticas), Saccharomyces (levaduras) y Rhodopseudomonas (bacterias fotosintéticas o fototróficas), redujeron la presencia de coliformes fecales, la demanda biológica de oxígeno, el contenido de nitratos y el $\mathrm{pH}$, entre otros cambios favorables (Fioravanti, Vega, \& Okumoto, 2005; Luna, 2006; Toc, 2012), que han hecho posible su evaluación y uso de forma generalizada en las localidades donde el tratamiento del agua es una estrategia esencial para preservar la salud.

En función de lo antes señalado, el objetivo de la presente investigación consistió en evaluar la eficiencia del uso de filtros de zeolitas combinados con el tratamiento de microorganismos eficaces $\left(E M \bullet 1^{\circledR}\right)$ y autóctonos de forma aislada en la corrección de las propiedades físicas de aguas para consumo humano procedentes de las localidades de Severino, Julián y Balsa en Medio del Ecuador.

\section{Materiales y métodos}




\section{Área objeto de la investigación}

Las muestras de agua provienen de la microcuenca hidrográfica del río Carrizal, ubicada entre las coordenadas $1^{\circ} 4^{\prime} 15.04^{\prime \prime}$ S, 79 52' 11.79" W, con un área de alrededor de $1390 \mathrm{~km}^{2}$. Se encuentra asentada en la provincia de Manabí y limita al norte con las cuencas del río Briceño y el río Jama; al sur con las cuencas del río Portoviejo y río Guayas; al este con el océano Pacífico y la cuenca del Estero Pajonal, y al oeste con la cuenca del río Guaya (MAE, 2009). Las comunidades estudiadas fueron Julián, Severino y Balsa en Medio (Figura 1). 
Tecnología y

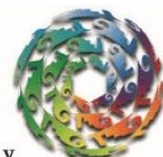

Ciencias
2020, Instituto Mexicano de Tecnología del Agua

Open Access bajo la licencia CC BY-NC-SA 4.0

(https://creativecommons.org/licenses/by-nc-sa/4.0/)

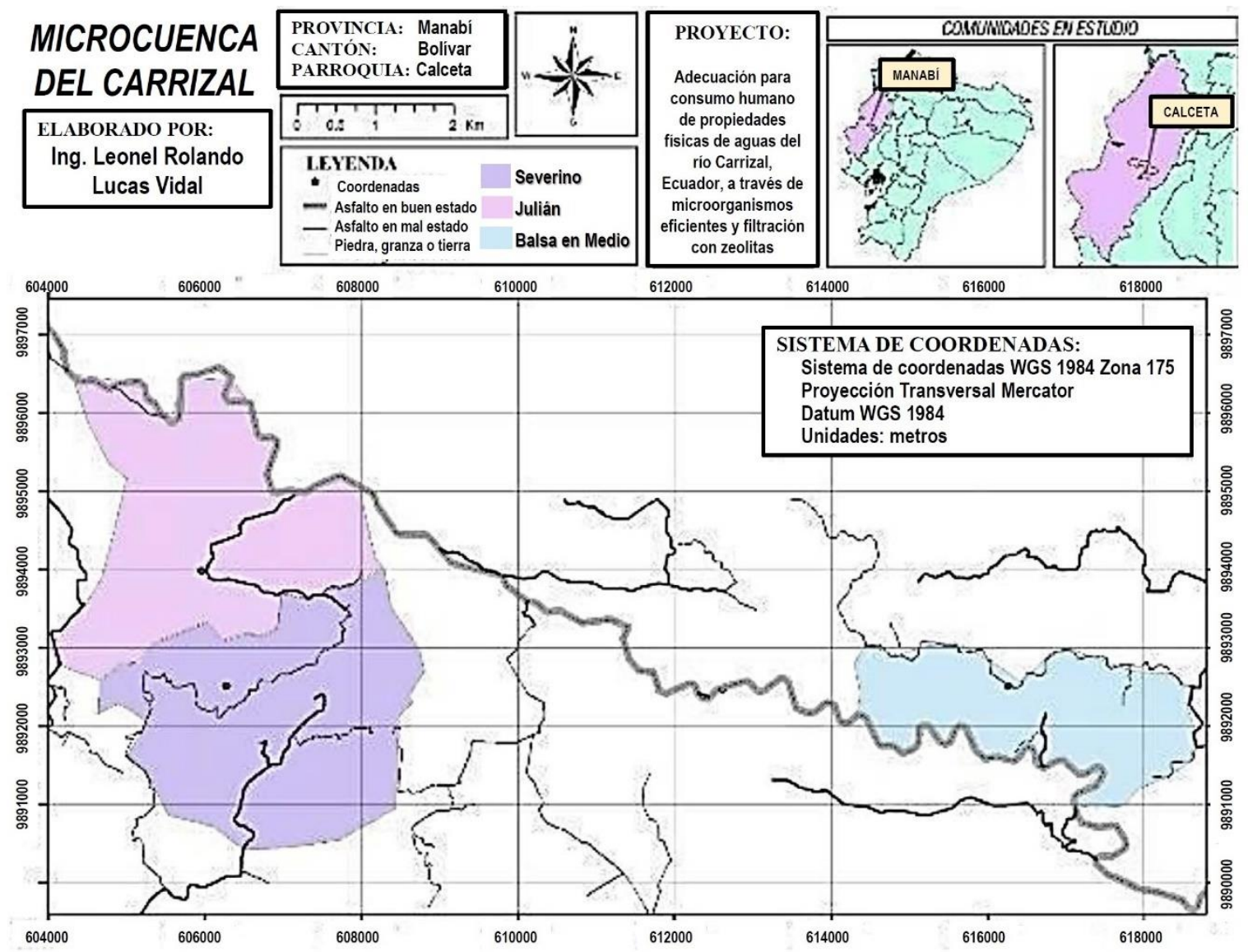

Figura 1. Ubicación relativa de las comunidades Julián, Severino y Balsa en Medio, provincia de Manabí, Ecuador.

\section{Tratamientos realizados al agua muestreada}

\section{Columnas de filtración de zeolitas}


Se evaluaron columnas de filtración de $5 \mathrm{~cm}$ de espesor, constituidas por zeolita cubana (mineral predominante: clinoptilolita) y zeolita ecuatoriana (mineral predominante: mordenita), contenidas en tubos de PVC de cuatro pulgadas de diámetro.

\section{Producto comercial EM•1 ${ }^{\circledR}$ (microorganismos eficaces)}

Para activar la formulación comercial se procedió de la siguiente manera: en un envase plástico sin contaminación química y limpio, con capacidad de dos litros, se colocaron $5 \mathrm{ml}$ de la formulación comercial $+5 \mathrm{ml}$ de melaza y se completó el volumen con $990 \mathrm{ml}$ de agua del grifo; el envase se cerró de manera hermética y se agitó manualmente durante 30 segundos, manteniéndose en ausencia de luz por seis días; se abrió a diario para dejar escapar los gases. Al finalizar este periodo se cuantificó el pH de la mezcla; en caso de que la muestra fuera superior a 3.5, se debía descartar, y en su defecto, prepararla de nuevo. La dosis del producto comercial correspondió al 5\% (AGEARTH, 2018).

Las muestras de agua consistieron de 5 I de agua incubados con $\mathrm{EM} \bullet 1^{\circledR}$, en la concentración de $5 \mathrm{ml} / \mathrm{l}$ durante $2 \mathrm{~h}, \mathrm{y}$ después pasadas por filtros constituidos por capas de $5 \mathrm{~cm}$ de zeolita, de origen cubano y ecuatoriano, respectivamente, contenidas en columnas de filtración. 


\section{Microorganismos autóctonos}

Los microorganismos autóctonos se extrajeron del mucílago del cacao (Saccharomyces cerevisiae), mucosa del intestino delgado de aves ponedoras (Bacillus spp.) y de camarón marino (Lactobacillus spp.), a través de fermentación. A continuación, se realizó el coctel utilizando como agua destilada y melaza al $5 \%$ para activarlos, se obtuvieron biopreparados para un litro con la concentración de Lactobacillus spp. 1.8 * $109 \mathrm{UFC} / \mathrm{ml}$ de Bacillus spp. 1.2 * 109 UFC/ml y Saccharomyces cerevisiae de $4.3 * 08 \mathrm{UFC} / \mathrm{ml}$.

De las muestras de agua colectadas se tomaron submuestras de 5 I de agua que fueron incubadas por dos horas con las dosis de 5, 10 y 15 $\mathrm{ml} / \mathrm{l}$ de los organismos autóctonos, respectivamente. Finalizado el periodo de incubación, se recolectaron muestras para los respectivos análisis físicos.

La manipulación, transporte, conservación y procedimientos analíticos de las muestras se llevaron a cabo con base en las recomendaciones de la APHA-AWWA-WEF (2012). La temperatura se evaluó por medio de un termómetro de mercurio para temperaturas máximas. La turbidez se determinó empleando un turbidímetro HANNA $2000 \mathrm{HI}$, calibrado con soluciones de formacina de 0 y 10 UFT (1 UFT = 1 UNT). La dureza total fue resultado del método de volumetría 
complejométrica, el cual utilizó ácido etilendiaminotetraacético (EDTA) como indicador. La determinación de sólidos totales se realizó a través del método gravimétrico, a partir de la evaporación y secado de la muestra a peso constante a $105{ }^{\circ} \mathrm{C}$; mientras que la concentración de color aparente del agua se obtuvo a través del método colorimétrico, con un espectrofotómetro DR $2800 \mathrm{HACH}$. Las determinaciones referidas se realizaron en el laboratorio de análisis químicos de la Escuela Superior Politécnica Agropecuaria de Manabí, ubicada en Manabí, Bolívar, Calceta, Ecuador.

\section{Tratamientos evaluados y diseño experimental}

Las vertientes se evaluaron como fuentes de agua y se representaron por el curso natural de agua que sirve de abastecimiento a la localidad, el agua almacenada en tanques de distribución en la comunidad y la de consumo colectada en los grifos de las casas.

Se tomaron cuatro (4) muestras de agua en cada una de las fuentes evaluadas para cada una de las localidades (Severino, Julián y Balsa en Medio), las cuales recibieron los siguientes tratamientos: 1) incubada con $\mathrm{EM} \bullet 1 \circledast$ a $5 \mathrm{ml} / \mathrm{l}$ y filtrada en zeolita cubana; 2) incubada con $\mathrm{EM} \bullet 1 \circledast$ a 5 $\mathrm{ml} / \mathrm{l}$ y filtrada en zeolita ecuatoriana; 3) incubada con $5 \mathrm{ml} / \mathrm{l}$; 4) $10 \mathrm{ml} / \mathrm{l}$ y 5-15 ml/l de microorganismos autóctonos, respectivamente. 
Se utilizó el diseño experimental de bloques al azar, con cuatro repeticiones. Previo al análisis de varianza (ANOVA), los valores de las características determinadas en las muestras de agua se examinaron por las pruebas de normalidad de Shapiro-Wilks y la de homogeneidad de varianza de Bartlett por el programa ASSISTAT (Silva \& Azevedo, 2016); Ios análisis de varianza se realizaron con el software InfoStat (Di Renzo et al., 2016). Por la naturaleza estructurada de los tratamientos, las comparaciones entre ellos se efectuaron por contrastes ortogonales y para determinar su significancia se empleó la prueba de $\mathrm{F}$ a $5 \%$ de probabilidad (Silva-Acuña, Álvarez, \& Silva-Acuña, 2000). La variable color no fue objeto de análisis estadístico, por no presentar variabilidad alguna entre las observaciones en las fuentes de muestreo y localidades.

\section{Resultados}

\section{Severino}

La Tabla 1 y la Tabla 2 exponen los resúmenes de los valores promedios y del análisis de varianza, respectivamente, para las variables 
Tecnología y

Ciencias $₫$ đAgua
2020, Instituto Mexicano de Tecnología del Agua

Open Access bajo la licencia CC BY-NC-SA 4.0

(https://creativecommons.org/licenses/by-nc-sa/4.0/)

temperatura, turbidez, sólidos totales y dureza en las localidades de Severino, Julián y Balsa en Medio.

Tabla 1. Valores promedios de las variables físicas: temperatura (TEM$\left.{ }^{\circ} \mathrm{C}\right)$; turbidez (TUR-NTU); sólidos totales (SOT-mg/dm³ $)$, y dureza total (DUR-mg/dm ${ }^{3}$ ), en cada una de las fuentes de agua muestreadas en las localidades de Severino, Julián y Balsa en Medio.

\begin{tabular}{|c|c|c|c|c|c|c|c|c|c|c|c|c|c|}
\hline \multirow{2}{*}{\multicolumn{2}{|c|}{$\begin{array}{c}\text { Tratamien- } \\
\text { tos }\end{array}$}} & \multicolumn{4}{|c|}{ Severino } & \multicolumn{4}{|c|}{ Julián } & \multicolumn{4}{|c|}{ Balsa en Medio } \\
\hline & & TEM & TUR & SOT & DUR & TEM & TUR & SOT & DUR & TEM & TUR & SOT & DUR \\
\hline \multirow{8}{*}{ 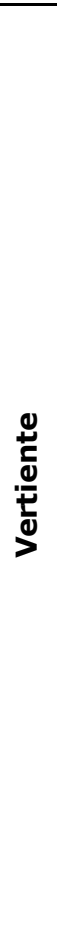 } & $\begin{array}{l}\mathrm{EM} \bullet 1+ \\
\mathrm{ZC}\end{array}$ & 28.48 & 8.5 & 140 & 138 & 27 & 8.5 & 283 & 133.1 & 28 & 9 & 386.3 & 140 \\
\hline & $\begin{array}{l}\mathrm{EM} \cdot 1+ \\
\mathrm{ZE}\end{array}$ & 27.18 & 3.75 & 126 & 150 & 25 & 5.5 & 247.5 & 132.5 & 28 & 5.75 & 298.8 & 145 \\
\hline & $\begin{array}{l}\ddot{x} E^{\circ} \mathbf{1}+ \\
Z\end{array}$ & 27.8 & 6.1 & 133 & 144 & 26 & 7 & 265 & 133 & 28 & 7.38 & 343 & 143 \\
\hline & $\begin{array}{l}\mathrm{MA}-5 \\
\mathrm{ml} / \mathrm{I}\end{array}$ & 26.98 & 3.25 & 125 & 150 & 25 & 5 & 246.3 & 133.7 & 28 & 5.5 & 290 & 143 \\
\hline & \begin{tabular}{|l}
$\mathrm{MA}-10$ \\
$\mathrm{ml} / \mathrm{l}$
\end{tabular} & 24.75 & 5.25 & 132 & 160 & 24 & 5 & 129 & 140.2 & 26 & 4.75 & 140 & 130 \\
\hline & $\begin{array}{l}\ddot{x} \text { MA } 5 \mathrm{y} \\
10 \mathrm{ml} / \mathrm{I}\end{array}$ & 25.9 & 4.3 & 129 & 155 & 25 & 5 & 188 & 137 & 27 & 5.13 & 215 & 137 \\
\hline & $\begin{array}{l}\mathrm{MA}-15 \\
\mathrm{ml} / \mathrm{l}\end{array}$ & 24.65 & 3.25 & 105 & 139 & 25 & 4.25 & 106.5 & 130.5 & 25 & 2.75 & 106 & 133 \\
\hline & $\ddot{\mathbf{x}}$ MA & 25.5 & 3.9 & 121 & 150 & 25 & 4.75 & 161 & 135 & 26 & 4.33 & 179 & 135 \\
\hline \multirow{2}{*}{$\begin{array}{l}0 \\
2 \\
\vec{J} \\
\frac{D}{\pi} \\
⺊\end{array}$} & $\begin{array}{l}\mathrm{EM} \cdot 1+ \\
\mathrm{ZC}\end{array}$ & 28.93 & 8.00 & 141 & 139 & 27 & 7.25 & 281 & 133.4 & 27 & 8.25 & 382.8 & 142 \\
\hline & $\begin{array}{l}\mathrm{EM} 1+ \\
\mathrm{ZE}\end{array}$ & 30.18 & 4.75 & 127 & 151 & 28 & 5.25 & 245 & 132.8 & 27 & 6 & 295.8 & 147 \\
\hline
\end{tabular}


Tecnología y

Ciencias Agua
2020, Instituto Mexicano de Tecnología del Agua

Open Access bajo la licencia CC BY-NC-SA 4.0

(https://creativecommons.org/licenses/by-nc-sa/4.0/)

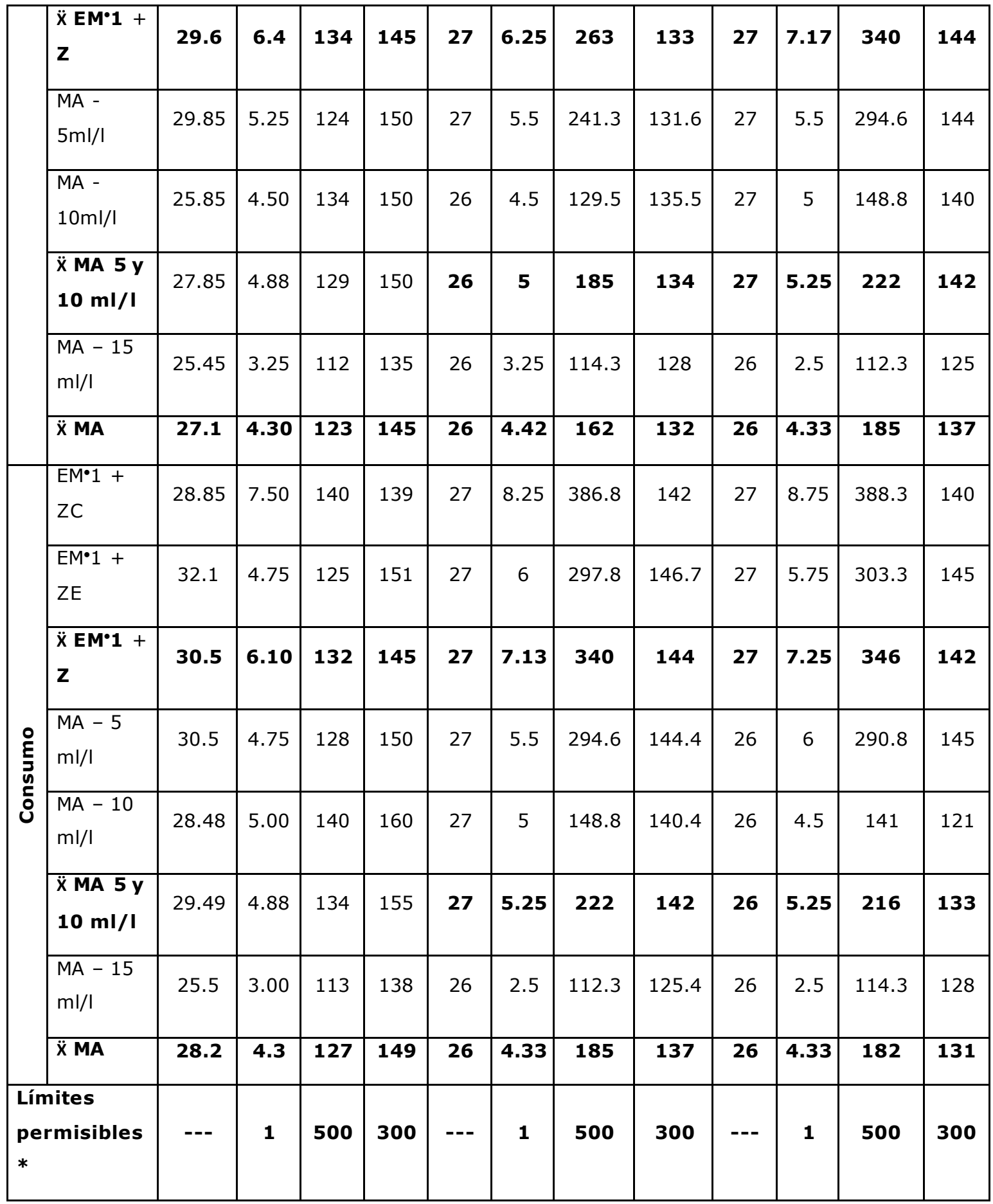

EM•1: microorganismos eficientes; MA: microorganismos autóctonos; Z: zeolitas; ZC: zeolita cubana; ZE: zeolita ecuatoriana; * NTE INEN (2014) y NTE INEN (2017). 
Tecnología y

Ciencias $\approx$ Agua
2020, Instituto Mexicano de Tecnología del Agua

Open Access bajo la licencia CC BY-NC-SA 4.0

(https://creativecommons.org/licenses/by-nc-sa/4.0/)

Tabla 2. Resumen del análisis de varianza para las variables físicas, temperatura (TEM), turbidez (TUR), sólidos totales (SOT) y dureza total

(DUR) en cada una de las fuentes de agua muestreadas en las localidades de Severino Julián y Balsa en Medio.

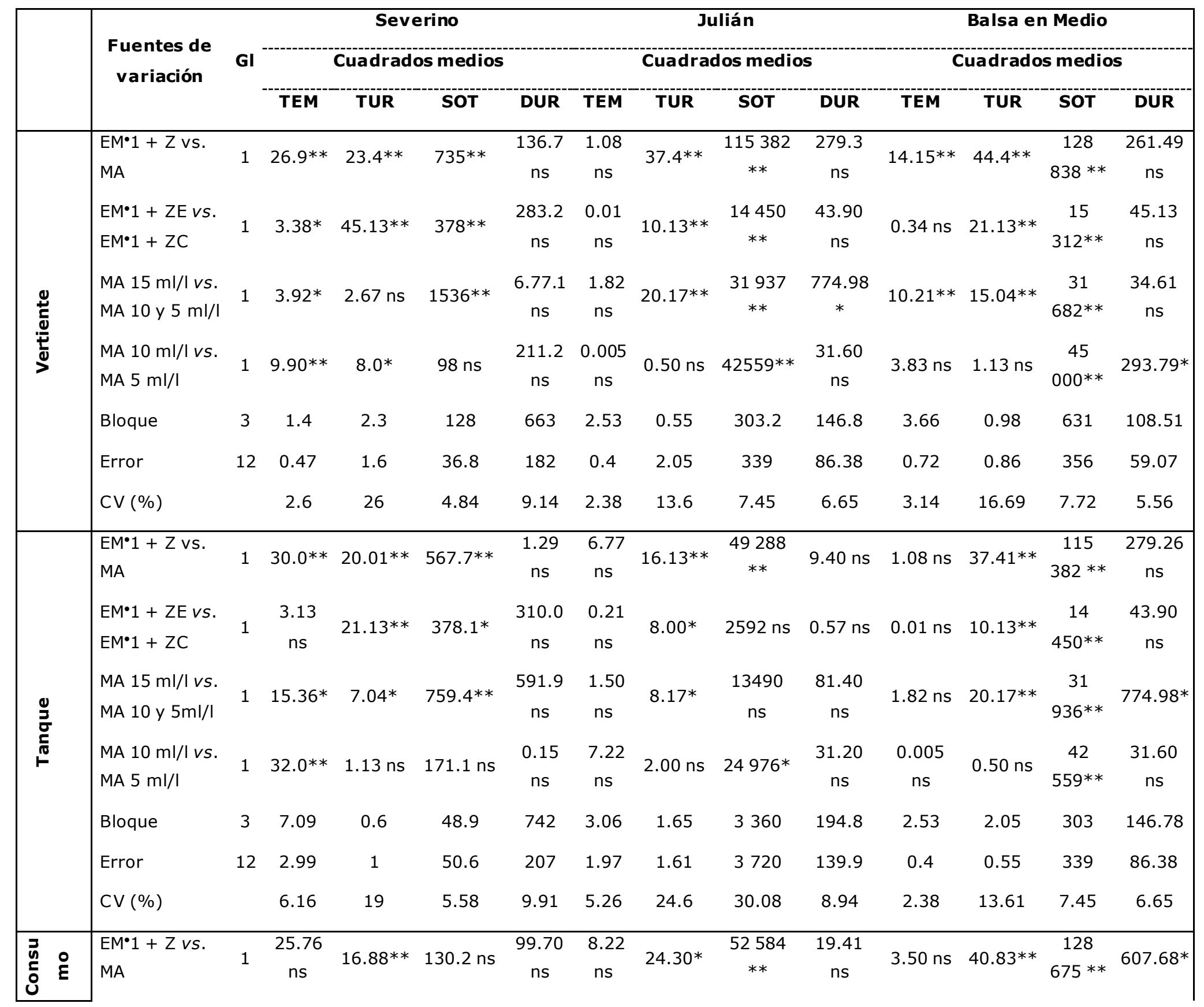


Tecnología y

Ciencias $\stackrel{\Xi}{\triangleleft}$ Agua
2020, Instituto Mexicano de Tecnología del Agua

Open Access bajo la licencia CC BY-NC-SA 4.0

(https://creativecommons.org/licenses/by-nc-sa/4.0/)

\begin{tabular}{|c|c|c|c|c|c|c|c|c|c|c|c|c|c|}
\hline $\begin{array}{l}\mathrm{EM} \cdot 1+\mathrm{ZE} v s \\
\mathrm{EM} \cdot 1+\mathrm{ZC}\end{array}$ & 1 & $\begin{array}{c}21.30 \\
\mathrm{~ns}\end{array}$ & $15.13^{* *}$ & $456.1 \mathrm{~ns}$ & $\begin{array}{c}318.0 \\
\mathrm{~ns}\end{array}$ & $\begin{array}{c}6.84 \\
\mathrm{~ns}\end{array}$ & $18.00 *$ & $2520 \mathrm{~ns}$ & $0.85 \mathrm{~ns}$ & $0.66 \mathrm{~ns}$ & $18.00 * *$ & $\begin{array}{c}14450 \\
* *\end{array}$ & $\begin{array}{c}43.25 \\
n s\end{array}$ \\
\hline $\begin{array}{l}\text { MA } 15 \mathrm{ml} / \mathrm{l} \text { vs. } \\
\text { MA } 10 \text { y } 5 \mathrm{ml} / \mathrm{l}\end{array}$ & 1 & $\begin{array}{c}42.40 \\
\mathrm{~ns}\end{array}$ & $9.38^{*}$ & $1120.7 * *$ & $\begin{array}{c}780.8 \\
n s\end{array}$ & $\begin{array}{l}0.57 \\
\mathrm{~ns}\end{array}$ & $12.50 *$ & $\begin{array}{c}17550 \\
\text { ns }\end{array}$ & $\begin{array}{c}112.93 \\
\text { ns }\end{array}$ & $0.57 \mathrm{~ns}$ & $20.17 * *$ & $\begin{array}{c}27557 \\
* *\end{array}$ & $\begin{array}{c}55.94 \\
\text { ns }\end{array}$ \\
\hline $\begin{array}{l}\text { MA } 10 \mathrm{ml} / / \mathrm{l} v \text {. } \\
\text { MA } 5 \mathrm{ml} / \mathrm{l}\end{array}$ & 1 & $\begin{array}{c}08.20 \\
\mathrm{~ns}\end{array}$ & $0.13 \mathrm{~ns}$ & $264.5 \mathrm{~ns}$ & $\begin{array}{c}174.4 \\
\text { ns }\end{array}$ & $\begin{array}{c}0.91 \\
\mathrm{~ns}\end{array}$ & $\begin{array}{c}0.001 \\
\mathrm{~ns}\end{array}$ & 27 495* & $84.76 \mathrm{~ns}$ & $\begin{array}{c}0.0012 \\
\mathrm{~ns}\end{array}$ & $4.50 \mathrm{~ns}$ & $\begin{array}{c}44888 \\
* *\end{array}$ & $\begin{array}{c}1161.6 \\
* *\end{array}$ \\
\hline Bloque & 3 & 47.35 & 0.5 & 10.1 & 1259 & 2.07 & 0.05 & 4525 & 118.5 & 1.60 & 0.60 & 418.75 & 203.95 \\
\hline Error & 12 & 9.17 & 1 & 118.1 & 180 & 1.93 & 2.72 & 3930 & 50.27 & 0.75 & 1.64 & 235 & 83.77 \\
\hline CV (\%) & & 10.41 & 21 & 8.42 & 9.09 & 5.49 & 29.2 & 30.97 & 5.29 & 3.28 & 23.3 & 6.19 & 6.75 \\
\hline
\end{tabular}

EM•1: microorganismos eficientes; MA: microorganismos autóctonos; Z: zeolitas; ZC: zeolita cubana; ZE: zeolita ecuatoriana; CV: coeficiente de variación. **: altamente significativo $(p<0.01)$; $*$ : significativo $(p<0.05)$; ns: no significativo.

En relación con la variable temperatura, se detectaron diferencias significativas $(p<0.05)$ entre los tratamientos a nivel de las muestras de la vertiente y del tanque; sin embargo, no hubo diferencias a nivel de las muestras de agua de consumo. Entre los EM•1 + zeolitas y los microorganismos autóctonos se detectaron diferencias significativas ( $p<$ 0.05), donde $\operatorname{los} \mathrm{EM} \bullet 1$ + zeolitas presentaron mayores temperaturas que los autóctonos.

Para las muestras de agua colectadas en la vertiente y la comparación entre las diferentes zeolitas usadas como filtros, se detectaron diferencias significativas ( $p<0.05)$, con mayor temperatura asociada con la zeolita cubana; a nivel de tanque no se detectaron diferencias entre las zeolitas. La comparación entre la dosis de $15 \mathrm{ml} / \mathrm{l}$ (más alta) de los microorganismos autóctonos versus las dosis de 10 y 5 $\mathrm{ml} / \mathrm{l}$ (menores) obtuvo diferencias significativas $(p<0.05)$ a nivel de vertiente y tanque. En ambos casos, las menores dosis presentan 
promedios de temperatura más elevados con relación con la dosis de 15 $\mathrm{ml} / \mathrm{l}$. Entre las dosis menores también se observaron diferencias, donde en ambos casos, la menor de las dosis $(5 \mathrm{ml} / \mathrm{l})$ presentó la temperatura más alta.

Para la variable turbidez se observaron diferencias significativas ( $p$ $<0.05$ ) en los tres tipos de fuentes de muestreo. Al comparar los organismos comercial eficiente, $\mathrm{EM} \bullet 1+$ zeolitas con los microorganismos autóctonos, en todos los casos, la menor turbidez estuvo asociada con los microorganismos autóctonos. La comparación entre la muestras tratadas con $\mathrm{EM} \bullet 1$ + zeolitas obtuvo diferencias significativas $(p<0.05)$, entre ellas, la zeolita ecuatoriana mostró menor turbidez. La comparación entre las dosis de los microorganismos autóctonos a nivel de tanque y consumo detectó diferencias significativas $(p<0.05)$ entre la dosis más alta y bajas, mientras que en la vertiente no hubo diferencias; la menor turbidez estuvo asociada con el tratamiento con $15 \mathrm{ml} / \mathrm{l}$. Entre las dosis menores, la comparación sólo fue significativa a nivel de la muestra proveniente de la vertiente, donde la menor turbidez ocurrió en el tratamiento con $5 \mathrm{ml} / \mathrm{l}$ del microorganismo autóctono.

En relación con la variable sólidos totales, la comparación entre los tratamientos $\mathrm{EM} \bullet 1$ + zeolitas y microorganismos autóctonos detectaron diferencias significativas $(p<0.05)$ en las muestras de la vertiente y del tanque, mas no así a nivel de consumo; en ambos casos, los sólidos totales están en menor proporción en las muestras tratadas con los microorganismos autóctonos. De manera similar a la comparación señalada antes, se detectaron diferencias a nivel de vertiente y tanque, al comparar los tipos de zeolitas; se constató que la zeolita ecuatoriana 
presentó el menor tenor de sólidos totales. En las tres muestras se distinguieron diferencias al comparar las dosis de los autóctonos; la dosis de $15 \mathrm{ml} / \mathrm{l}$ fue la que presentó el menor nivel de sólidos totales, y entre las dosis de 10 y $5 \mathrm{ml} / \mathrm{l}$ no se observaron diferencias entre ellas en todos los niveles de muestreo.

La variable física dureza presentó un comportamiento particular: no presentó diferencias significativas entre los tratamientos en todos los niveles de muestreo.

\section{Julián}

En el caso de la localidad de Julián destaca que la variable temperatura no mostró diferencias significativas $(p<0.05)$ entre comparaciones de tratamientos en ninguno de los puntos de muestreo evaluados. En el caso de la variable turbidez, la comparación inicial entre EM•1 + zeolitas y los microorganismos autóctonos detectó diferencias significativas $(p<0.05)$ en todos los niveles de muestreo; los menores tenores de la variable están asociados con los microorganismos autóctonos. Al comparar los tipos de zeolitas en todos los casos, se obtuvo diferencia significativa con menor turbidez relacionada con la zeolita ecuatoriana. Las comparaciones entre dosis de microorganismos autóctonos sólo detectaron diferencias significativas $(p<0.05)$ a nivel de vertiente y del tanque, donde los menores valores están vinculados con la dosis de $15 \mathrm{ml} / \mathrm{l}$; mientras que 
al comparar entre las dosis menores no se observó diferencia significativa alguna.

Para la variable sólidos totales, la comparación entre los tratamientos $\mathrm{EM} \bullet 1$ + zeolitas y los microorganismos autóctonos arrojó diferencias significativas $(p<0.05)$ en todas las muestras evaluadas; los menores valores están relacionados con los microorganismos autóctonos. Entre los tipos de zeolitas, las diferencias significativas $(p<0.05)$ se observaron sólo a nivel de vertiente, donde los menores valores de SOT se asocian con la zeolita ecuatoriana. De la misma manera se detectaron a nivel de vertiente diferencias significativas $(p<0.05)$ entre las dosis de microorganismos autóctonos, donde los menores tenores corresponden a la dosis de $15 \mathrm{ml} / \mathrm{l}$; entre las dosis menores, las diferencias significativas $(p<0.05)$ presentes en todos los niveles de muestreo señalan que la dosis de $10 \mathrm{ml} / \mathrm{l}$ tiene los valores menores de SOT.

Para la variable dureza sólo se observó diferencia significativa a nivel de la muestra, proveniente de la vertiente, únicamente en la comparación entre la dosis alta de microorganismos autóctonos y las más bajas, siendo que los menores valores de dureza se relacionan con la dosis de $15 \mathrm{ml} / \mathrm{l}$.

\section{Balsa en Medio}


Para la temperatura sólo se obtuvieron diferencias significativas ( $p<$ 0.05) a nivel de vertiente. En la comparación de los microrganismos eficientes (comerciales) $\left(\mathrm{EM}^{\bullet} 1+\right.$ zeolitas) en relación con los microorganismos autóctonos, las diferencias indican que las mayores temperaturas estuvieron relacionadas con $\mathrm{EM} \bullet 1+$ zeolitas. No se detectaron distinciones entre las zeolitas utilizadas; sin embargo, ocurrieron diferencias significativas $(p<0.05)$ entre la dosis mayor y las menores de los autóctonos, donde las mayores temperaturas se vincularon con las menores dosis (10 y $5 \mathrm{ml} / \mathrm{l}$ ) y entre ellas no se detectan diferencias significativas.

En la variable turbidez se lograron diferencias significativas ( $p<$ 0.05 ) en los tres fuentes de agua muestreados. La comparación entre la combinación EM•1 + zeolitas con los microorganismos autóctonos para todos los casos indica que la menor turbidez se asoció con los autóctonos. Entre los tipos de zeolitas en todas las fuentes de muestreo existieron diferencias significativas, coincidiendo la menor turbidez asociada con la zeolita ecuatoriana. Entre las dosis de los autóctonos, donde también hubo diferencias significativas $(p<0.05)$ en todas las muestras, se constató menor turbidez con la dosis de $15 \mathrm{ml} / \mathrm{l}$; mientras que entre las dosis menores no se detectaron diferencias.

Para los sólidos totales se detectaron diferencias significativas ( $p<$ 0.05 ) en todos los niveles de muestreo para todos los tratamientos evaluados. Entre los microorganismos comerciales eficientes, combinados con filtrado en zeolitas y los microorganismos autóctonos, las diferencias señalan que los valores menores de SOT estuvieron vinculados con los autóctonos. En la comparación entre las zeolitas para todos los casos, las 
proporciones menores de sólidos totales estuvieron asociadas con la zeolita ecuatoriana. Dentro de las dosis de los autóctonos para todos los casos, los valores menores de la variable se relacionan con la dosis de 15 $\mathrm{ml} / \mathrm{l}$. Entre las dosis mínimos, los menores tenores de sólidos totales están relacionados con la dosis de $10 \mathrm{ml} / \mathrm{l}$.

Para la dureza del agua, la comparación de los tratamientos con EM•1 + zeolitas y los microrganismos autóctonos sólo obtuvo diferencias significativas $(p<0.05)$ a nivel de consumo, donde los menores tenores de dureza se vinculan con los autóctonos. Entre las zeolitas no hubo diferencias significativas en ninguna de las muestras; sin embargo, en la comparación entre las dosis de los autóctonos sólo se detectó diferencias a nivel del tanque, donde el tratamiento con $15 \mathrm{ml} / \mathrm{l}$ presentó el menor tenor de dureza. Entre las dosis menores de los autóctonos se identificaron diferencias a nivel de la vertiente y de consumo, en el que los menores tenores de dureza correspondieron a las dosis de $10 \mathrm{ml} / \mathrm{l}$.

\section{Discusión}

En términos generales, en todas las localidades, las variables físicas evaluadas cumplen con las normativas aplicables a las condiciones de calidad de agua de consumo y cuerpos de agua dulce vigentes en Ecuador, estipuladas en las normas NTE INEN (2014) (Agua potable para consumo 
Tecnología y

Ciencias $₫$ Agua
2020, Instituto Mexicano de Tecnología del Agua

Open Access bajo la licencia CC BY-NC-SA 4.0

(https://creativecommons.org/licenses/by-nc-sa/4.0/)

humano) y NTE INEN (2017) (Agua purificada envasada y agua purificada mineralizada envasada), con excepción de la variable turbidez, que excede los límites permisibles en todas las localidades y en las fuentes de agua muestreadas.

En el caso de las variables temperatura, sólidos totales y dureza, se obtuvo una tendencia clara en el mejoramiento de las propiedades del agua cuando se utilizan microorganismos autóctonos, y en segundo lugar está el empleo de la zeolita ecuatoriana como material filtrante. En cuanto a la eficiencia de las dosis de microorganismos autóctonos, la tendencia fue en orden decreciente de las dosis, donde la de $15 \mathrm{ml} / \mathrm{l}$ resultó ser la más eficiente. La variable color presentó valores $<25$ en todas las cuantificaciones en el nivel de localidades y fuentes de muestreo, razón por la cual no fue posible realizar ningún análisis estadístico ni, en consecuencia, hacer inferencias estadísticas con sus resultados.

La variable temperatura presentó poca variación bajo las condiciones evaluadas, con valores que oscilan entre 25 y $30^{\circ} \mathrm{C}$, sin alteraciones superiores a $5^{\circ} \mathrm{C}$, como consecuencia de los tratamientos aplicados, lo cual se ajusta a lo recomendado por Mejía (2015). Estos valores oscilan de igual forma en los valores de las temperaturas ambientales de la zona de experimentación, ubicada dentro de la franja tropical, lo cual es consistente con los resultados obtenidos por Vera et al. (2016) para zonas áridas.

Los sólidos totales están asociados con las sales disueltas en una muestra de agua después de la remoción de sólidos suspendidos. El rango de variación obtenido, después de los tratamientos, se ubicó entre 100 y $141 \mathrm{mg} / \mathrm{dm}^{3}$, muy por debajo de los valores establecidos por la norma 
NTE INEN (2017). Baque-Mite et al. (2016) encontraron valores dentro de los rangos de aceptabilidad de calidad ambiental para el cantón Quevedo, provincia de Los Ríos, Ecuador.

La dureza está determinada por el contenido de carbonatos, bicarbonatos, cloruros, sulfatos, y ocasionalmente nitratos de calcio y magnesio. Por lo general provoca sabor indeseable al agua potable. Debido a los bajos niveles de dureza derivados de los tratamientos, que variaron entre 130 y $160 \mathrm{mg} / \mathrm{dm}^{3}$, se puede considerar que el agua tratada se clasifica como suave; esto es beneficioso, pues su tratamiento no representa altos costos ni genera problemas por incrustaciones en las tuberías de distribución, como lo indican Gutiérrez, Scull y Oramas (2006). Los valores fueron inferiores al límite permisible de $300 \mathrm{mg} / \mathrm{dm}^{3}$. Estos resultados coinciden con los obtenidos por Puglla (2017) para la microcuenca Yaguaimi en Ecuador.

La turbidez representa la capacidad del material suspendido en el agua para impedir el paso de la luz, causada por la presencia de material coloidal en suspensión; puede ser de origen orgánico o inorgánico, como arcilla, materia orgánica e inorgánica, plancton y microorganismos. Los valores del producto de los tratamientos fluctuaron entre 3 y 8.5 unidades nefelométricas de turbidez y superaron los límites permisibles de 1 NTU.

La Figura 2 muestra la comparación de los tratamientos evaluados en la corrección de la turbidez del agua de las diferentes fuentes; destaca que ninguno de los tratamientos logró corregir esta variable a los límites permisibles de la norma NTE INEN (2017). Los mejores resultados se lograron con la aplicación de microorganismos autóctonos en dosis de 15 $\mathrm{ml} / \mathrm{l}$ (Contraste 3), aplicados a nivel de tanque y de consumo, que 
Tecnología y

Ciencias $\approx$ Agua
2020, Instituto Mexicano de Tecnología del Agua

Open Access bajo la licencia CC BY-NC-SA 4.0

(https://creativecommons.org/licenses/by-nc-sa/4.0/)

redujeron los niveles de turbidez a valores cercanos a 2 NTU, independientemente de la localidad evaluada.
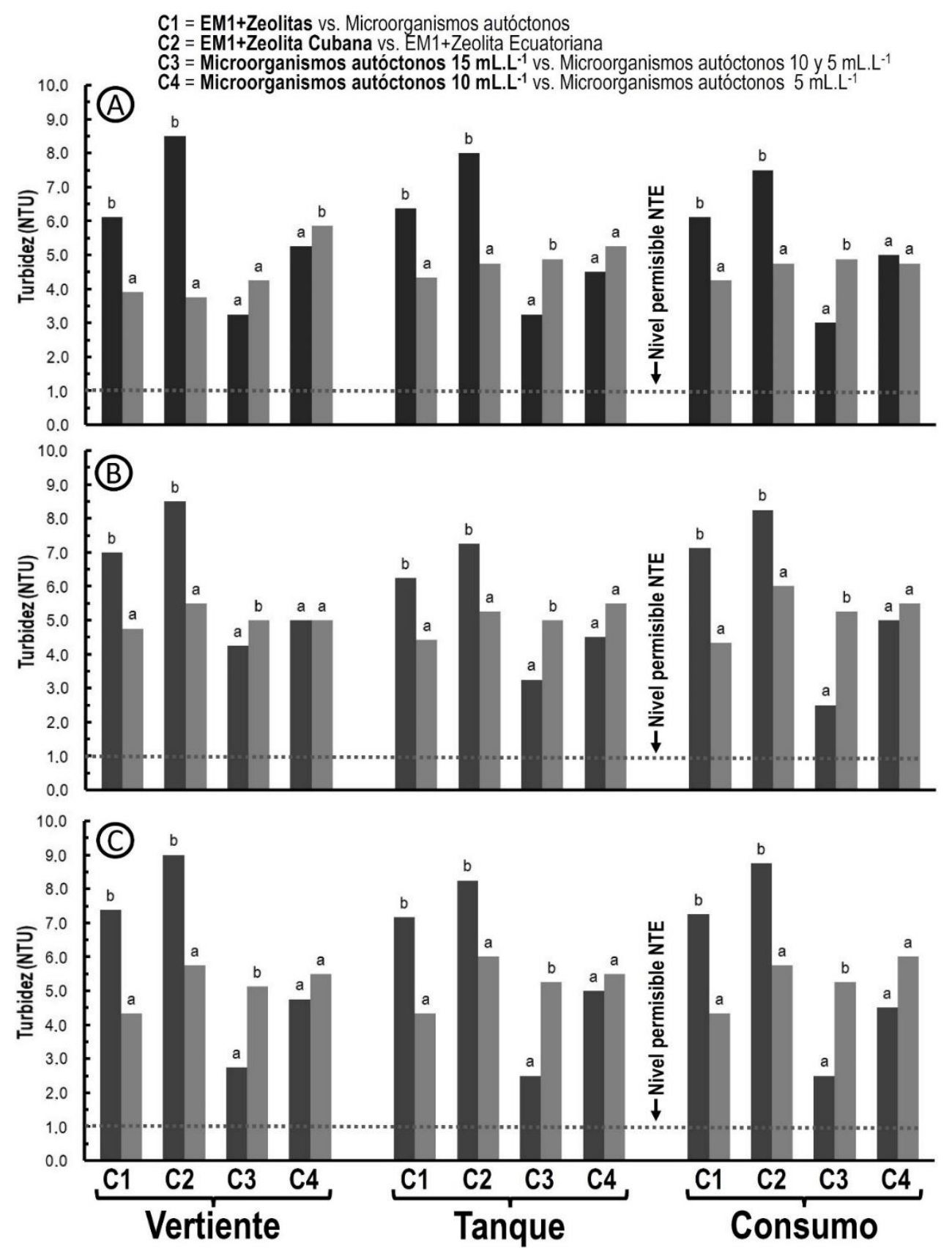
Figura 2. Promedio de los contrastes ( $\mathrm{C} 1, \mathrm{C} 2$, $\mathrm{C} 3$ y $\mathrm{C} 4)$ para la variable turbidez en cada una de las fuentes muestreadas en las localidades de Severino (A), Julián (B) y Balsa en Medio (C).

Estos resultados podrían estar asociados con el tiempo entre la aplicación de los tratamientos y el momento de ejecución de las evaluaciones. Marco, Azario, Metzler y García (2004) señalan que las partículas mayores a un micrón sedimentan de forma espontánea, mientras que la velocidad de sedimentación de las partículas pequeñas (menores al micrón de diámetro) es muy baja, por lo que requieren tratamiento para lograrla en tiempos útiles.

Varios autores han encontrado que la turbidez tiene una fuerte correlación positiva con la cantidad de coliformes totales tanto a la entrada como a la salida de los filtros (Marco et al., 2004; Hoff \& Geldreich, 1981; Barrenechea, 2004). Por su parte, Power y Nagy (1999) encontraron que con turbidez baja (alrededor de 1 UNT) no se percibe influencia sobre la frecuencia de coliformes observados en placas.

Los resultados obtenidos de la turbidez en esta investigación concuerdan con los de Marco et al. (2004), que evidenciaron la existencia de fallas en las etapas de clarificación y desinfección del proceso de potabilización del agua, conseguida a partir del río Uruguay y del arroyo Molino, para el abastecimiento de agua potable en las ciudades. Por otro lado, Marco et al. (2004) señalan que es recomendable incluir la turbidez entre las determinaciones mínimas o básicas de mayor frecuencia para el monitoreo de la calidad del agua potable captadas por fuentes superficiales, incluso en el caso de pequeñas y medianas ciudades, dada 
la rapidez de la obtención del resultado y el bajo costo en su determinación.

Tradicionalmente, la eliminación de la turbidez se realiza mediante procesos de coagulación, asentamiento y filtración. Desde el punto de vista de tratamientos orgánicos, Parra et al. (2011) demostraron la efectividad del mucílago extraído de Opuntia wentiana en la clarificación de aguas de alta turbidez (100-200 UNT), lo que indica la posibilidad de emplearlo como coagulante primario en las plantas de tratamiento de aguas potables, con valores de remoción superiores a $70 \%$.

El empleo de microorganismos eficientes con propósitos de reducción de turbidez ha sido poco estudiada. Luna (2006) señala que los protozoarios son los principales consumidores de las poblaciones bacterianas en los sistemas acuáticos e intervienen en la formación de flóculos sedimentables. La presencia de protozoarios en sistemas de depuración biológica ayuda de modo significativo a la mejora de la calidad de los efluentes, reduciendo la DBO, turbidez del medio y cantidad de bacterias. De manera similar, Canales y Sevilla (2017) encontraron mejoras significativas en la reducción de la turbidez superiores al 95\%, en un medio colonizado por $\mathrm{EM}^{\circledR}$, relacionado con la competencia por los nutrimentos, la liberación de sustancias enzimáticas que secuestran minerales o vitaminas necesarias para el crecimiento, o de los patógenos que lo impiden. También señalan que los microorganismos eficientes transforman la materia orgánica, al liberar y sintetizar sustancias, y compuestos como aminoácidos, enzimas, vitaminas, sustancias bioactivas, hormonas y minerales solubles, que convierten aguas residuales domésticas en aguas aptas para su vertido o utilización. 


\section{Conclusiones}

1. El tratamiento con microorganismos autóctonos en dosis de $15 \mathrm{ml} / \mathrm{l}$ y el tratamiento de filtración con zeolita ecuatoriana asociado con el microorganismo eficiente $(E M \bullet 1)$ generaron ventajas significativas para las variables turbidez, sólidos totales y dureza, con independencia de la localidad y de la fuente de agua tratada.

2. La variable temperatura presentó mayores valores relacionados con los tratamientos con los organismos eficientes $\left(\mathrm{EM}^{\bullet} 1\right)$ cuando son filtrados con la zeolita cubana y, de manera similar, con la dosis de $5 \mathrm{ml} / \mathrm{l}$ de los microorganismos autóctonos.

3. Las variables físicas de las localidades de Severino, Julián y Balsa en Medio cumplen con la normatividad aplicable a las condiciones de calidad del agua de consumo y cuerpos de agua dulce vigentes en Ecuador.

4. Los niveles de turbidez del agua de la microcuenca del río Carrizal exceden los niveles permisibles para el consumo humano. Los tratamientos aplicados no lograron la corrección a los límites reglamentados.

\section{Referencias}


APHA-AWWA-WEF, American Paint Horse Association, American Water Works Association, Water Environment Federation. (2012). Standard Methods for the Examination of Water and Waste Water (22 ${ }^{\text {nd }}$ ed.) Washington: American Public Health Association.

AGEARTH, Asociación de Graduados de la EARTH-Ecuador. (2018). Desplegable informativo sobre los microorganismos eficaces. Riobamba, Ecuador: Asociación de Graduados de la EARTH. Escuela Superior Politécnica de Chimborazo.

Baque-Mite, R., Simba-Ochoa, L., González-Ozorio, B., Suatunce, P., Diaz-Ocampo, E., \& Cadme-Arevalo, L. (2016) Calidad del agua destinada al consumo humano en un cantón de Ecuador. Revista Ciencia UNEMI, 9 (20), 109-117.

Barrenechea, A. (2004). Tratamiento de agua para consumo humano. Plantas de infiltración rápida. Manual I: Teoría. Tomo I. Lima, Perú: Centro Panamericano de Ingeniería Sanitaria y Ciencias del Ambiente.

Canales, H., \& Sevilla, A. (2017). Evaluación del uso de microorganismos eficaces en el tratamiento de efluentes domésticos residuales del distrito de Patapo (tesis de grado). Escuela Profesional de Ingenieńa Química, Universidad Nacional "Pedro Ruiz Gallo", Lambayeque, Perú.

Covarrubias, C., García, R., Yánez, J. , \& Arriagada, R. (2008). Preparation of CPB-modified FAU zeolite for the removal of tannery waste water contaminants. Journal of Porous Materials, 15(4), 491-498. 
Cuchimaque, C., Vargas, L. Y., \& Ríos, C. (2013). Remoción de Fe y Mn en aguas naturales por adsorción-oxidación sobre clinoptilolita. Revista de la Facultad de Ingeniería Universidad de Antioquia, 66, 24-44.

Di Renzo, J. A., Casanoves, F., Balzarini, M. G., González, L., Tablada, M., \& Robledo C. W. (2016). InfoStat versión 2016. Córdoba, Argentina: Grupo InfoStat, FCA, Universidad Nacional de Córdoba.

Fioravanti, N., Vega, C., \& Okumoto, J. (2005). Eficiencia de los microorganismos eficaces (EM) en la estabilización de lodos sépticos para su uso agrícola. Revista de la Universidad EARTH. Tierra Tropical, 1(1), 69-76.

Global Health and Education Foundation, National Academy of Science. (2007). El agua potable segura es esencial. Recuperado de https://www.koshland-science-

museum.org/water/html/es/Treatment/Household-Water-

Treatment-Systems.html

Gutiérrez, O., Scull, I., \& Oramas, A. (2006). Zeolita natural para la reducción de la dureza del agua. Revista Cubana de Ciencia Agrícola, 40(2), 191-192.

Hoff, J. \& Geldreich, E. (1981). Comparison of the biocidal efficiency of alternative desinfectans. Journal American Water Works Association, 73, 40.

Luna, P. D. M. (2006). Atlas de ciliados y otros microorganismos frecuentes en sistemas de tratamiento aerobio de aguas residuales. 
Facultad de Química. México, DF, México: Universidad Nacional Autónoma de México. .

Marco, L., Azario, R., Metzler, C., \& García, M. (2004). La turbidez como indicador básico de calidad de aguas potabilizadoras a partir de fuentes superficiales. Higiene y Sanidad Ambiental, 4, 72-82.

Márquez, E., Herrera, T. N., \& Gutiérrez, N. M. (2000) Características físico-químicas de las zeolitas naturales como medio filtrante. Congreso Interamericano de Ingeniería Sanitaria y Ambiental. Porto Alegre, Brasil.

Mejía, M. (2015). Análisis de la calidad del agua para consumo humano y percepción local de las tecnologías apropiadas para su desinfección a escala domiciliaria, en la microcuenca El Limón, San Jerónimo, Honduras (trabajo de grado). Centro Agronómico Tropical de Investigación y Enseñanza, Costa Rica.

MAE, Ministerio del Ambiente de Ecuador. (2009). Proyecto Adaptación al Cambio Climático a través de una Efectiva Gobernabilidad del Agua en el Ecuador (PACC). Estudio de vulnerabilidad actual a los riesgos climáticos en el sectorde los recursos hídricos en las cuencas de los Ríos Paute, Jubones, Catamayo, Chone, Portoviejo y Babahoyo. Quito, Ecuador: Manthra Editores.

Mishué, E., Hernández, M., \& González, J. (2012). Remoción de arsénico de agua para uso y consumo humano empleando arcillas y zeolitas mediante diferentes materiales de adsorción (tesis de maestría). Universidad Nacional Autónoma de México, México, DF, México. 
NTE INEM. Norma Técnica Ecuatoriana, Instituto Nacional Ecuatoriano de Nomalización. (2014). ISO 1108. Agua potable. Requisitos. s.I.: Norma Técnica Ecuatoriana. Guayas, Ecuador: Instituto Nacional Ecuatoriano de Nomalización. Recuperado de http://www.pudeleco.com/files/a16057d.pdf

NTE INEN. Norma Técnica Ecuatoriana, Instituto Nacional Ecuatoriano de Nomalización. (2017). ISO 2200. Agua purificada envasada. Requisitos. s.I.: Norma Técnica Ecuatoriana. Guayas, Ecuador: Instituto Nacional Ecuatoriano de Nomalización. Recuperado de https://www.normalizacion.gob.ec/buzon/normas/nte_inen_22002.pdf

Orellana, J. (2015). Características del agua potable. Ingeniería Sanitaria. Ingeniería Sanitaria-UTN-FRRO. Recuperado de https://www.frro.utn.edu.ar/repositorio/catedras/civil/ing_sanitari a/Ingenieria_Sanitaria_A4_Capitulo_03_Caracteristicas_del_Agua_ Potable.pdf

Paredes, D. (2014). Estudio de la modificación termoquímica de una zeolita para incrementar su capacidad de intercambio catiónico (CIC) y aplicarla en la remediación de aguas contaminadas con Plomo (II). http://repositorio.unsa.edu.pe/handle/UNSA/2923.

Parra, Y., Cedeño, M., García, M., Mendoza, I., González, Y., \& Fuentes, L. (2011). Clarificación de aguas de alta turbidez empleando el mucílago de Opuntia wentiana (Britton \& Rose)/(Cactaceae). Redieluz, 1 (1), 27-33.

Power, K., \& Nagy, L. (1999). Relationship between bacterial regrowth and some physical and chemical parameters within Sydney's 
drinking water distribution system. Water Research, 33(3), 741750.

Puglla, L. J. (2017). Evaluación de la calidad del agua cruda captada con fines de consumo humano en la parroquia Patuca y propuesta de un plan de manejo de la microcuenca Yaguaimi (trabajo de grado). Escuela de Ingeniería Ambiental, Universidad de Cuenca, Cuenca, Ecuador.

Silva, F., \& Azevedo C. (2016). The Assistat Software Version 7.7 and its use in the analysis of experimental data. African Journal of Agricultural Research, 11(39), 3733-3740.

Silva-Acuña, R. Álvarez, V. H., \& Silva-Acuña, A. (2000). Cómo comparar correctamente tratamientos de naturaliza cualitativa. Agronomía Tropical, 50(2), 151-155.

Toc, M. (2012). Efecto de los microorganismos eficientes (ME) en las aguas residuales de la granja porcina de Zamorano, Honduras (trabajo de grado). Facultad de Ciencia y Producción Agrícola, Escuela Agrícola Panamericana, Zamorano, Honduras.

Vera, I. L., Rojas, M., Chávez, W., \& Arriaza, B. T. (2016) Evaluación de materiales filtrantes para el reúso en agricultura de aguas residuales tratadas provenientes de zonas áridas. Ciencia e Ingeniería Neogranadina, 26(1).

Wang, S. \& Peng, Y. (2010). Natural zeolites as effective adsorbents in water and wastewater treatment. Chemical Engineering Journal, $156,11-24$. 